\title{
Collision kernel and interatomic potential
}

\author{
Tak-San Ho and Shih-I Chu \\ Department of Chemistry, University of Kansas, Lawrence, Kansas 66045
}

(Received 10 December 1985)

\begin{abstract}
We present a detailed study of the influence of the form and strength of the interatomic potential on the one-dimensional elastic collision kernel $W\left(v_{z}^{\prime}, v_{z}\right)$, a quantity of interest in the study of the effects of velocity-changing collisions on laser spectroscopic line shapes. We find that the absolute magnitudes of collision kernels are very sensitive while normalized collision kernels are moderately sensitive to the potential form used. This indicates the importance of employing realistic interatomic potentials and reliable differential cross sections in the accurate determination of collision kernels. For the case of the Lennard-Jones $(12,6)$ potential, we found a universal semiclassical Lennard-Jones (SCLJ) analytical model function, which is the combination of a semiclassical expression for small to medium scattering angles and a classical expression for large scattering angles, capable of providing correct average quantum-mechanical behaviors of differential cross sections for all scattering angles. This greatly facilitates the (often time-consuming) numerical evaluation of the collision kernel integrals and exhibits the correct collision kernel line profiles. It is found that the SCLJ collision kernel consists of a strongly peaked forward diffractive zone (small-angle scatterings), reflecting the nature of velocity resonance, as well as a broad wing region due to large-angle scatterings. Ambiguities associated with the drawbacks of the hard-sphere model, the small-angle classical long-range model, and the classical Lennard-Jones model are analyzed and clarified. While our analysis is confined to the $\mathrm{Na}-\mathrm{Ar}$ and $\mathrm{Ar}-\mathrm{Ar}$ systems, the conclusions derived from this study are general and are expected to be also applicable to other systems where both the long- and short-range interactions play essential roles in velocity-changing collisions.
\end{abstract}

\section{INTRODUCTION}

The study of the effects of velocity-changing collisions ${ }^{1}$ on laser spectroscopic line shapes is a subject of much current interest both experimentally and theoretically. Various nonlinear optical techniques ${ }^{2-9}$ such as saturation spectroscopy, two-photon spectroscopy, velocityselective optical pumping, stimulated photon echo, etc., have been applied to investigate the velocity-changing effects of collisions between active atoms and rare-gas perturbers. The quantity of interest here is the collision kernel $W\left(v^{\prime}, v\right)$ describing the probability per unit time that a collision will change the projection of the atomic velocity on the laser-beam axis from $v^{\prime}$ to $v$.

Several theoretical modelings on the collision kernels have been proposed and scrutinized. ${ }^{2,4,5,7,9}$ The phenomenological Keilson and Storer (KS) collision kernel, ${ }^{10-12}$ having the appearance of a displaced Gaussian form, is by far the most commonly adopted model. The KS kernel provides analytically tractable solutions for the line shape and yields a thermal distribution of atomic velocities in the limit when a large number of collisions take place. However, it is best applied only to the situations where the active atoms are much heavier than the perturbers and at high pressures. ${ }^{2,4}$ Improvement over the KS model can be in principle achieved by taking into account the dependence of velocity-changing collisions upon the strength and the form of the interaction potential between the active atom and the perturber atom. Introduction of realistic potentials, however, usually leads to intractable analytical expressions for the collision kernels.
For example, even for the simplest case of the hard-sphere (HS) interaction model, the expression for the collision kernel is rather cumbersome. ${ }^{4}$ Nevertheless, the classical hard-sphere (CHS) kernel has been found to be superior to the KS kernel as the former gives a better description of the effects of large-angle scattering for heavy perturbers and at lower pressures. ${ }^{4}$ As will be shown later, however, the CHS model tends to overestimate the contribution from the large-angle scatterings caused by the repulsive wall, and is liable to break down when attractive counterpart of interatomic potential plays a significant role. Indeed, the CHS model fails to reproduce the relatively small velocity changes contributing in the vicinity of the velocity resonance. ${ }^{6}$

Rigorously speaking, the profile of the collision kernel should reflect collisional information from all scattering angles. It consists mainly of contributions from the forward diffractive zone (small-angle scatterings), exhibiting the nature of the velocity resonance, as well as a broad wing region due to large-angle scatterings. To describe such an uncharacteristic structure of the collision kernel in a unified fashion, one needs to invoke more realistic interatomic potential than the HS model and carry out the corresponding differential cross sections in a more exact manner. The purpose of this article is to exploit the sensitivity of the differential cross section and the collision kernel with respect to the strength and the form of interatomic potentials and thereby clarify many ambiguities currently existing in the subject. For simplicity, we shall confine our discussion to the case of elastic population kernels where only one interatomic potential is involved. 


\section{COLLISION KERNEL AND DIFFERENTIAL CROSS SECTION}

\section{A. Collision kernel}

The (three-dimensional) elastic collision kernel $W\left(\mathbf{v}^{\prime}, \mathbf{v}\right)$, representing the probability density per unit time that a collision changes the active-atom velocity from $\mathbf{v}^{\prime}$ to $\mathbf{v}$, is related to the differential cross section by the following formal expression: ${ }^{5}$

$$
\begin{aligned}
W\left(\mathbf{v}^{\prime}, \mathbf{v}\right)=N\left(\frac{m}{\mu}\right)^{3} \int d \mathbf{v}_{r}^{\prime} \int & d \mathbf{v}_{r} W_{p}\left(\mathbf{v}^{\prime}-\mathbf{v}_{r}^{\prime}\right) \\
& \times \delta\left[\mathbf{v}_{r}-\mathbf{v}_{r}^{\prime}-\frac{m}{\mu}\left(\mathbf{v}-\mathbf{v}^{\prime}\right)\right] \\
& \times v_{r}^{-1} \delta\left(v_{r}-v_{r}^{\prime}\right) \\
& \times \frac{d \sigma}{d \Omega}\left(v_{r}^{\prime},\left|\mathbf{v}_{r}-\mathbf{v}_{r^{\prime}}\right|\right) .
\end{aligned}
$$

Equation (1) represents the collision kernel in the centerof-mass frame averaged over the perturber velocity distribution $W_{p}$ consistent with conservation of momentum and energy. Here $N$ is the density of perturbers; $m$ and $\mu$ are, respectively, the mass of the active atom and the reduced mass of the system $\left[\mu=m m_{p} /\left(m+m_{p}\right), m_{p}\right.$ the mass of the perturber], $\mathbf{v}_{r}^{\prime}=\mathbf{v}^{\prime}-\mathbf{v}_{p}^{\prime}$ is the relative velocity of the active atom $\left(\mathbf{v}^{\prime}\right)$ and the perturber $\left(\mathbf{v}_{p}^{\prime}\right)$ before the collision, and $v_{r}$ is the relative velocity after the collision. The perturber velocity (thermal) distribution is given by

$$
W_{p}\left(v_{p}\right)=\left(\pi u_{p}^{2}\right)^{-3 / 2} \exp \left(-v_{p}^{2} / u_{p}^{2}\right),
$$

where $u_{p}$ is the most probable speed of the perturber. The quantity of the laser spectroscopic importance is the onedimensional kernel, $W\left(v_{z}^{\prime}, v_{z}\right)$, representing the probability per unit time that a collision changes the projection of the active-atom velocity on the laser-beam direction from $v_{z}^{\prime}$ to $v_{z}$. The one-dimensional kernel is obtained by averaging the three-dimensional kernel $W\left(\mathbf{v}^{\prime}, \mathbf{v}\right)$ over the transverse-velocity components $\left(\mathbf{v}_{t}^{\prime}, \mathbf{v}_{t}\right)$ of the active atoms, namely,

$$
W\left(v_{z}^{\prime}, v_{z}\right)=\int W\left(\mathbf{v}^{\prime}, \mathbf{v}\right) \rho\left(\mathbf{v}_{t}^{\prime}\right) d \mathbf{v}_{t}^{\prime} d \mathbf{v}_{t},
$$

where

$$
\rho\left(v_{t}\right)=\left(\pi u^{2}\right)^{-1} \exp \left(-v_{t}^{2} / u^{2}\right)
$$

is the thermal distribution of the transverse speed $v_{t}$ and $u$ is the most probably active-atom speed. Equation (3) can be reduced to a triple integral of the form ${ }^{5}$

$$
W\left(v_{z}^{\prime}, v_{z}\right)=4 N \pi^{-1 / 2} \beta k \int_{0}^{\infty} d s_{t} \int_{0}^{\infty} d q \int_{-\infty}^{\infty} d p e^{-\beta^{2}(p-y)^{2}} \exp \left[-k^{-1}\left(q^{2}+q_{0}^{2}\right)\right] \frac{d \sigma}{d \Omega}\left[\left(q_{0}^{2}+p^{2}+q^{2}\right)^{1 / 2}, \eta\right],
$$

where

$$
\begin{aligned}
& \beta=u / u_{p}=\left(m_{p} / m\right)^{1 / 2}, \\
& k=\left(1+\beta^{2}\right) / \beta^{2}, \\
& \boldsymbol{\eta}=\frac{m}{\mu}\left(\mathbf{v}-\mathbf{v}^{\prime}\right), \\
& \mathbf{S}=\left(\mathbf{v}-\mathbf{v}^{\prime}\right) / u=\mathbf{S}_{t}+S_{\mathbf{z}} \hat{z}, \\
& q_{0}^{2}=S_{t}^{-2}\left(\frac{1}{2} k S^{2}+p S_{z}\right)^{2},
\end{aligned}
$$

and

$$
y=v_{z}^{\prime} / u
$$

Note that the elastic differential cross section $(d \sigma / d \Omega)$ depends upon $\eta$ and $v_{r}=\left|\mathbf{v}_{r}\right|=u\left(q_{0}^{2}+q^{2}+p^{2}\right)^{1 / 2}$, and the scattering angle $\theta$ in the center-of-mass frame is related to $\eta$ and $v_{r}$ by the relation

$$
\theta=2 \sin ^{-1}\left(\eta / 2 v_{r}\right) \text {. }
$$

\section{B. Differential cross section}

In the quantum-mechanical treatment ${ }^{13}$ of the scattering process for central potentials $V(r)$, the differential cross section is given by

$$
d \sigma / d \Omega=|f(\theta)|^{2},
$$

where $f(\theta)$ is the scattering amplitude which can be evaluated via the Rayleigh sum over partial waves:

$$
f(\theta)=(2 i k)^{-1} \sum_{l=0}^{\infty}(2 l+1)\left(e^{2 i \delta_{l}}-1\right) P_{l}(\cos \theta)
$$

with $\delta_{l}$ the phase shift, $l$ the orbital angular momentum quantum number, $k\left(=\mu v_{r}\right)$ the initial wave number, and $P_{l}(\cos \theta)$ the Legendre polynomial. The phase shifts $\delta_{l}$ are usually determined by numerical integration of the radial wave equation

$$
\frac{d^{2} \chi_{l}(r)}{d r^{2}}+\left(k^{2}-\frac{2 \mu}{\hbar^{2}} V(r)-\frac{l(l+1)}{r^{2}}\right) \chi_{l}(r)=0
$$

subject to the asymptotic form 


$$
\chi_{l}(r) \sim \sin \left(k r-l \pi / 2+\delta_{l}\right)
$$

Such a procedure, while providing accurate results for $(d \sigma / d \Omega)$, is not practical for the evaluation of the collision kernel as all together it involves a great amount of computational work. A better strategy is to obtain some approximate analytical forms for $(d \sigma / d \Omega)$ valid for various energy $(k)$ and angular $(\theta)$ regions. In the following we discuss some of the most commonly used interatomic potentials in laser spectroscopy.

\section{Lennard-Jones $(12,6)$ potential}

The Lennard-Jones $(12,6)$ potential is

$$
V(r)=\epsilon\left[\left(\frac{r_{m}}{r}\right)^{12}-2\left(\frac{r_{m}}{r}\right)^{6}\right],
$$

where $\epsilon$ is the potential well depth and $r_{m}$ is the radius of potential minimum.

(a) The classical small-angle scattering approximation ${ }^{14}$ is

$$
\begin{aligned}
\left.\int \frac{d \sigma}{d \Omega}\right]^{\mathrm{CLJ}}(\theta)= & \frac{r_{m}^{2}}{24[100-(154 / \pi) K \theta]^{1 / 2}}\left[\frac{3 \pi}{4}\right]^{1 / 3} \theta^{-4 / 3} K^{-1 / 3} \\
& \times\left(\left\{10+[100-(154 / \pi) K \theta]^{1 / 2}\right\}^{4 / 3}+\left\{10-[100-(154 / \pi) K \theta]^{1 / 2}\right\}^{4 / 3}\right), \text { at } \theta<\theta_{r}=50 \pi / 77 K,
\end{aligned}
$$

where $\theta_{r}$ is the rainbow angle, $K=E / \epsilon$ is the reduced energy with $E=1 / 2 \mu v_{r}^{2}$, and CLJ denotes classical Lennard-Jones.

(b) The classical large-angle scattering approximation ${ }^{14}$ is

$$
\begin{aligned}
&\left(\frac{d \sigma}{d \Omega}\right)^{\mathrm{CLJ}}(\theta)=\frac{r_{m}^{2}}{(2.222158)^{2}} K^{-1 / 6} \\
& \text { at } \theta>\theta_{r}=50 \pi / 77 K .
\end{aligned}
$$

Equation (11a) is obtained in the first-order momentum approximation and is determined by the attractive branch of the deflection function only. This formula shows the monotonic decrease of the differential cross section at small angles (large impact parameters) $(d \sigma / d \Omega) \sim \theta^{-7 / 3}$ followed by the rainbow singularity at $\theta=\theta_{r}$. This classical expression also diverges as $\theta$ goes to zero. Equation (11b) is an approximate form for the differential cross section at large angles (small impact parameters) determined by the repulsive part of the potential only. It is nearly angle independent and it depends only weakly on the reduced energy $K$.

\section{Purely long-range potential of the form $V(r)=-C_{6} r^{-6}$, with $C_{6}=2 \epsilon r_{m}^{6}$}

(a) The classical (CL) small-angle scattering approximation $^{9,13}$

$$
\left(\frac{d \sigma}{d \Omega}\right]^{\mathrm{CL}}(\theta)=r_{m}^{2} \frac{5}{6}\left[\frac{3 \pi}{16}(2 \epsilon) / K\right]^{1 / 3} \theta^{-7 / 3} .
$$

Similar to Eq. (11a), this expression shows $\theta^{-7 / 3}$ angular dependence for small angles and diverges at $\theta=0$.

(b) Semiclassical (SCL) small-angle scattering approximation. The singularity at the forward $(\theta=0)$ direction for classical expressions such as Eq. (12) can be removed if one uses a semiclassical description of the differential cross section. A convenient semiempirical model function for a small-angle differential cross section has been recently obtained by Beijerinck et al., ${ }^{15}$

$$
\begin{array}{r}
\left(\frac{d \sigma}{d \Omega}\right)^{\mathrm{SCL}}(\theta)=\left(\frac{d \sigma}{d \Omega}\right)^{\mathrm{SCL}}(0)\left[1-C_{1} \sin \left(C_{2} \theta^{* 2}\right)\right. \\
\left.+C_{3} \theta^{* 2}\right]^{-7 / 6}, \\
0 \leq \theta^{*} \leq 3.9 .
\end{array}
$$

Here $\left\{C_{i}\right\}$ is a universal set of parameters given by $C_{1}=3.75, C_{2}=0.556$, and $C_{3}=2.94$. The reduced angle $\theta^{*}$ and the differential cross section at the forward $(\theta=0)$ direction, $(d \sigma / d \Omega)^{\mathrm{SCL}}(0)$, are described in Ref. 15. The asymptotic behavior of this function for $\theta^{*} \ll 1$ and $\theta^{*} \gg 1$ is in good agreement with the corresponding semiclassical and classical results. As will be shown below, Eq. (13) provides a good universal approximation for both small and intermediate angles.

\section{Hard-sphere potential}

This has been discussed extensively in the literature., 4 The collision kernels can be worked out analytically for both the quantum-mechanical (small-angle) ${ }^{5}$ and the classical (all-angle) ${ }^{4}$ scatterings.

\section{A unified expression of $(d \sigma / d \Omega)$ for all angles}

A proper account of the collision-kernel line profile requires the information of differential cross sections at all angles. To facilitate the numerical evaluation of the collision kernel for realistic potentials, it is expedient to have a universal analytical expression $(d \sigma / d \Omega)$ valid for the whole angular range. While such an expression is not available for any realistic potential (excluding the hardsphere model), we found (as will be demonstrated below) 
the combination of the semiclassical expression, Eq. (13), with the classical large-angle asymptotic expression, Eq. (11b), in fact provides an excellent approximation to the quantum mechanical scattering of the $\mathrm{LJ}(12,6)$ potential at all angles. We shall denote such a unified expression by the notation $(d \sigma / d \Omega)^{\mathrm{SCLJ}}(\theta)$.

\section{CASE STUDIES: Na-Ar AND Ar-Ar SYSTEMS}

To investigate the quality of various approximate differential cross-section expressions and the sensitivity of collision kernels with respect to the form and strength of interatomic potentials, we shall consider the elastic collisions between $\mathrm{Na}$ and $\mathrm{Ar}$ and between $\mathrm{Ar}$ and $\mathrm{Ar}$ in their respective electronic ground states. These represent interesting intermediate cases (active-atom-perturber mass ratio $\sim 1$ ) where both the attractive and the repulsive parts of the interaction potentials are significant in the determination of the collision kernels. We note that while we shall consider $\mathrm{Na}$ as the active atom in the $\mathrm{Na}$ Ar collision, the way of deciding which collision partner is the active atom or perturber poses no practical significance in our analysis below. We shall assume both the binary collision approximation and the impact approximation are valid. The $\mathbf{L J}(12,6)$ potential parameters are shown in Table I.

\section{A. Differential cross-section calculations}

For the Na-Ar system, we shall assume the thermal temperature $T=300 \mathrm{~K}$. This corresponds to a reduced energy approximately equal to 3 . The elastic differential cross sections as a function of the scattering angle $\theta$ are shown in Fig. 1. In Fig. 1(a), the quantum-mechanical results $^{16}(d \sigma / d \Omega)^{\mathrm{QMLJ}}$, i.e., from Eqs. (6) and (7), for a Lennard-Jones $(12,6)$ potential are presented along with the results from the unified expression $(d \sigma / d \Omega)^{\mathrm{SCLJ}}(\theta)$. Apart from the neglect of the rainbow and orbiting effects, the semiclassical expression, Eq. (13), provides an excellent representation of the average behavior of the quantum-mechanical results for small and intermediate angles. The quantum-mechanical rainbow oscillations are in fact not significant here as they tend to be averaged out by the thermal averaging calculations in collision-kernel integrals. The differential cross sections at large angles are also described well by the classical expression, Eq. (11b), as can be seen by their good agreement with the QMLJ results. Thus the unified expression $(d \sigma / d \Omega)^{\operatorname{SCLJ}}(\theta)$ appears to be able to provide a reasonably good representation for the whole angular range. Figure 1(b) depicts $(d \sigma / d \Omega)^{\mathrm{CL}}(\theta), \quad(d \sigma / d \Omega)^{\mathrm{CL}}(\theta)$,

TABLE I. Potential parameters of the Lennard-Jones interatomic potential $V(r)=\epsilon\left[\left(r_{m} / r\right)^{12}-2\left(r_{m} / r\right)^{6}\right]$.

\begin{tabular}{lll}
\hline \hline System & \multicolumn{1}{c}{$\epsilon$ (a.u.) } & $r_{m}$ (a.u.) \\
\hline $\mathrm{Na}-\mathrm{Ar}^{\mathrm{a}}$ & $1.95 \times 10^{-4}$ & 9.5 \\
$\mathrm{Ar}^{\mathrm{A}} \mathrm{Ar}^{\mathrm{b}}$ & $4.48185 \times 10^{-4}$ & 7.0978 \\
\hline \hline
\end{tabular}

${ }^{\mathrm{a}}$ Reference 17.

${ }^{\mathrm{b}}$ Reference 18. $(d \sigma / d \Omega)^{\mathrm{QMHS}}(\theta)$, and $(d \sigma / d \Omega)^{\mathrm{CHS}}(\theta)$, standing for the classical Lennard-Jones, Eqs. (11a) and (11b); classical (small-angle) long-range; Eq. (12), quantum-mechanical (small-angle) hard-sphere; and classical hard-sphere results, respectively. In general one sees that $(d \sigma / d \Omega)^{\mathrm{CLJ}}(\theta)$ gives good agreement with $(d \sigma / d \Omega)^{\mathrm{QMLJ}}$ except at the classical rainbow angle and at the forward direction $(\theta=0)$ where the former diverges. $(d \sigma / d \Omega)^{\mathrm{CL}}$ provides good results at small angles (except near $\theta=0$ where it diverges) but fails at large angles. While the classical hard-sphere results agree well with the quantum mechanical results of a more realistic $\mathrm{LJ}$ potential at large angles, they fail completely at small angles. In particular, one notes that owing to the lack of the attractive part of the potential, the forward scattering amplitudes of the hard-sphere models could be many orders of magnitude
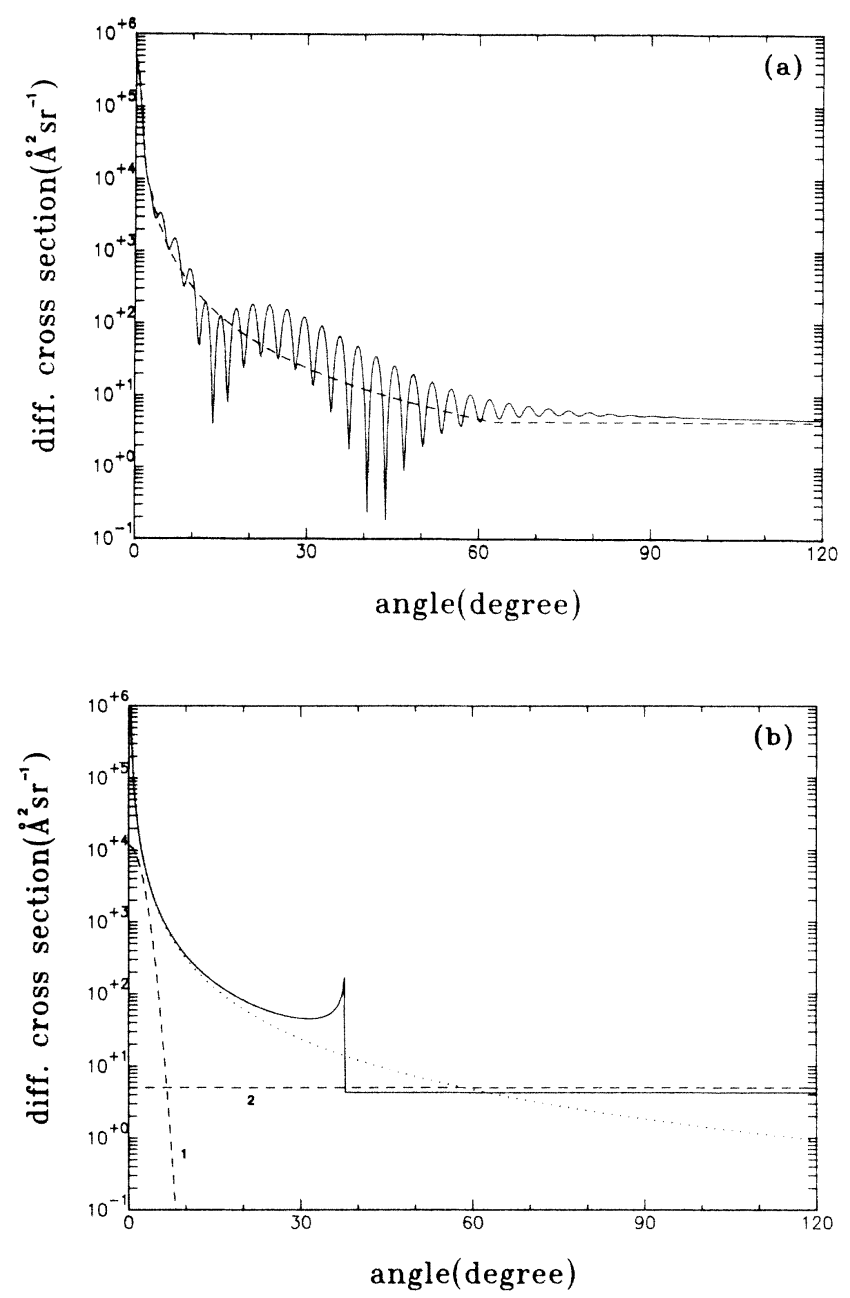

FIG. 1. Elastic differential cross sections $(d \sigma / d \Omega)(\theta)$ as functions of the scattering angle $\theta$ in the center-of-mass frame for the Na-Ar system. The relative velocity $v_{r}^{\prime}$ is set to be equal to the most probable active-atom (Na) speed $u=\left(2 k_{B} T / m_{\mathrm{Na}}\right)^{1 / 2}$ at temperature $T=300 \mathrm{~K}$. (a) Solid curve, $(d \sigma / d \Omega)^{\mathrm{QMLJ}}(\theta)$; dashed curve, $(d \sigma / d \Omega)^{\mathrm{SCLJ}}(\theta)$. (b) Solid curve, $(d \sigma / d \Omega)^{\mathrm{CLJ}}(\theta)$; dotted curve, $(d \sigma / d \Omega)^{\mathrm{CL}}$; dashed curve $1,(d \sigma / d \Omega)^{\mathrm{QMHS}}(\theta)$ at small angles; dashed curve $2,(d \sigma / d \Omega)^{\mathrm{CHS}}(\theta)$. The hard-sphere radius is taken to be $2^{-1 / 6} r_{m}$. 
smaller than the correct values. The discrepancies in the differential cross sections in the forward direction result in much difference (in absolute magnitude) in the collision-kernel line profiles in the diffractive zone.

In Fig. 2, we show the elastic differential cross sections for the $\mathrm{Ar}-\mathrm{Ar}$ system. The thermal temperature is assumed to be $T=600 \mathrm{~K}$. This amounts to approximately a reduced energy $K \approx 2$. Good agreements again are found between $(d \sigma / d \Omega)^{\mathrm{QML}}(\theta)$ and $(d \sigma / d \Omega)^{\operatorname{SCL}}(\theta)$. Previous conclusions for $\mathrm{Na}-\mathrm{Ar}$ about the behaviors of $(d \sigma / d \Omega)^{\mathrm{CLJ}}(\theta),(d \sigma / d \Omega)^{\mathrm{CL}}(\theta),(d \sigma / d \Omega)^{\mathrm{QMHS}}(\theta)$, and $(d \sigma / d \Omega)^{\mathrm{CHS}}(\theta)$ also hold for $\mathrm{Ar}$-Ar case. The $\mathrm{LJ}(12,6)$ parameters for Ar-Ar are listed in Table I. Note that the asymmetric system $\mathrm{Na}-\mathrm{Ar}$ has somewhat shallower potential well than the symmetric Ar-Ar system. Neither system, however, represents the extreme case of $\left(m_{p} / m\right)<1$ or $\left(m_{p} / m\right) \gg 1$. The behavior of either extreme case is somewhat easier to predict and will not be discussed in this work.
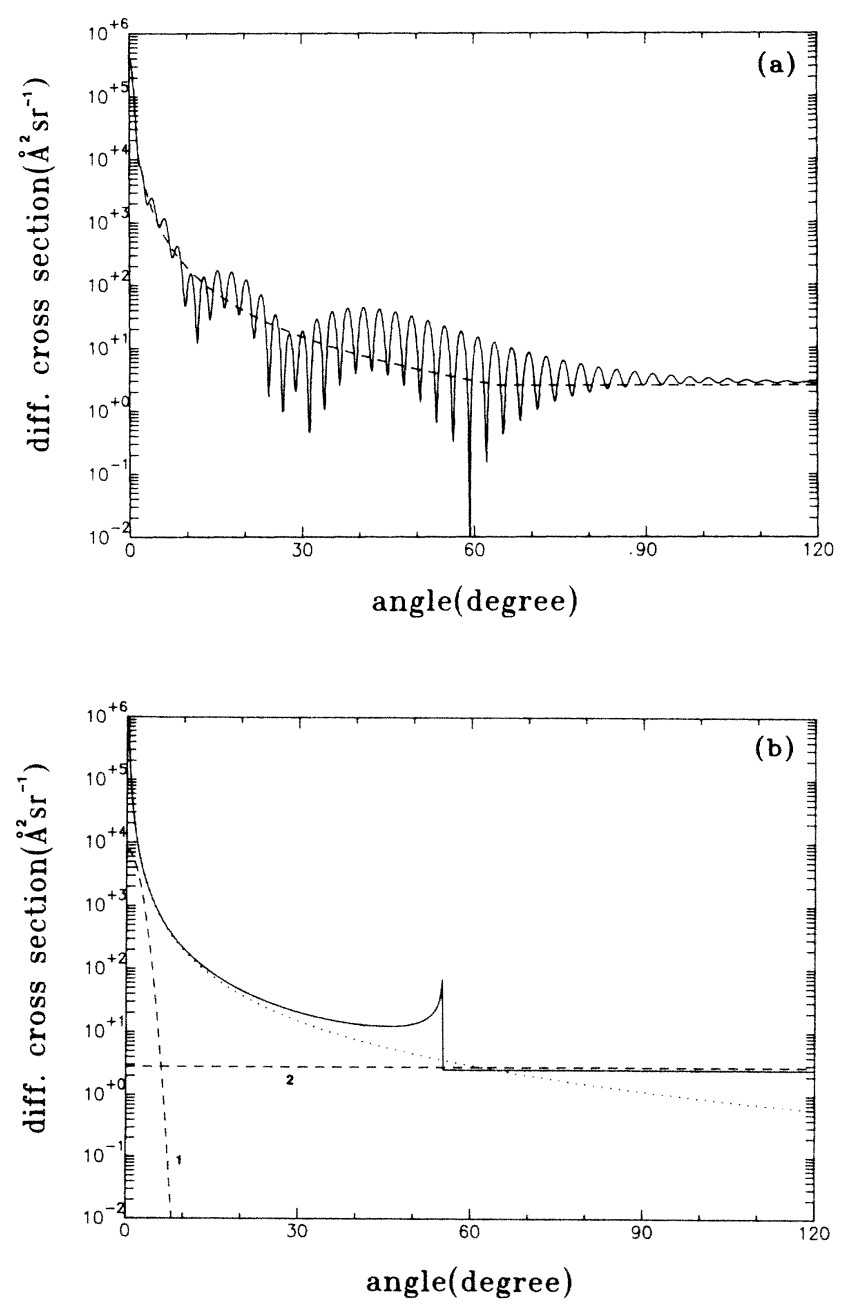

FIG. 2. $(d \sigma / d \Omega)(\theta)$ vs $\theta$ for the Ar-Ar system at $v_{r}^{\prime}=u=\left(2 k_{B} T / m_{A r}\right)^{1 / 2}$ at $T=600 \mathrm{~K}$. Notations same as Fig. 1.

\section{B. Collision-kernel calculations}

The hard-sphere model is the only case where the onedimensional collision kernel, Eq. (5), can be worked out analytically. ${ }^{4,5}$ Figure 3(a) shows the results from a classical hard-sphere (CHS) model ${ }^{4}$ and a quantum mechanical small-angle hard-sphere (QMSAHS) model ${ }^{5}$ for the $\mathrm{Na}$-Ar collision. The normalized kernel $W\left(v_{z}^{\prime}, v_{z}\right)$ is presented as a function of $v_{z}$ at three different initial $v_{z}^{\prime}$, viz., $v_{z}^{\prime}=0, u$, and $2 u$, where $u=\left(2 k_{B} T / m_{\mathrm{Na}}\right)^{1 / 2}$ is the most probable active atom $(\mathrm{Na})$ velocity at temperature $T$ $\left(=300 \mathrm{~K}\right.$ in this case). It is seen that $W\left(v_{z}^{\prime}, v_{z}\right)$ for the CHS case exhibits a broad structure around $v_{z}=v_{z}^{\prime}$. When $v_{z}^{\prime}=0$, the collision kernel is symmetric about $v_{z}=v_{z}^{\prime}$, whereas it is rather asymmetric when $v_{z}^{\prime} \neq 0$; the asymmetry grows with $v_{z}^{\prime}$ as shown. The broad structure is mainly due to the large angle scattering from the repulsive wall. When $v_{z}^{\prime}$ moves farther away from zero, the number
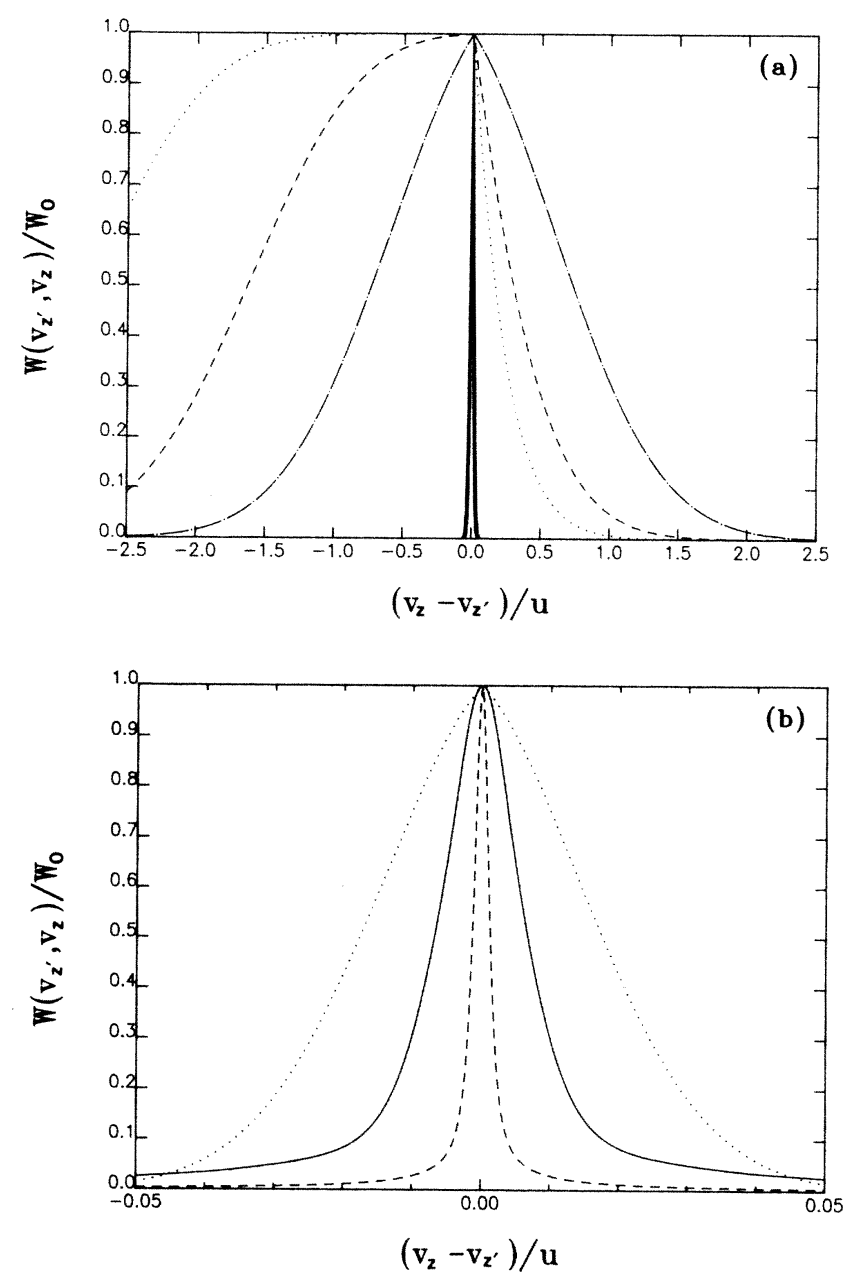

FIG. 3. Normalized one-dimensional collision kernels $W\left(v_{z}^{\prime}, v_{z}\right) / W_{0}$ as functions of $\left(v_{z}-v_{z}^{\prime}\right) / u$ for the Na-Ar system at $T=300 \mathrm{~K}$, where $W_{0}=W\left(v_{z}^{\prime}, v_{z}=v_{z}^{\prime}\right)$ and $u=\left(2 k_{B} T / m_{\mathrm{Na}}\right)^{1 / 2}$. (a) Solid curves, small-angle QMHS results (the three curves corresponding to $v_{z}^{\prime}=0, u$, and $2 u$ are indistinguishable in this scale); dashed-dotted curve, CHS at $v_{z}^{\prime}=0$; dashed curve, CHS at $v_{z}^{\prime}=u$; and dotted curve, CHS at $v_{z}^{\prime}=2 u$. (b) Solid curve, SCLJ data at $v_{z}^{\prime}=0$; dashed curve, CLJ data at $v_{z}^{\prime}=0$; dotted curve, small-angle QMHS data at $v_{z}^{\prime}=0$. 
of atoms scattered with $v_{z}<v_{z}^{\prime}$, i.e., those deflected with larger angles, becomes more, and thus causes larger asymmetry in $W\left(v_{z}^{\prime}, v_{z}\right)$. On the other hand, the small-angle quantum-mechanical results predict an exactly symmetrical, but much narrower profile (about $v_{z}=v_{z}^{\prime}$ ) compared to the CHS model, and the width becomes narrower as $v_{z}^{\prime}$ increases. Both hard-sphere calculations fail to predict the forward diffractive zone (small-angle scattering) behavior of $W\left(v_{z}^{\prime}, v_{z}\right)$ in the region around $v_{z}=v_{z}^{\prime}$, due to the neglect of the attractive part of the potential. Nevertheless, the broad wing profile, as to be seen later, of $W\left(v_{z}^{\prime}, v_{z}\right)$ is correctly predicted because it mainly comes from large-angle deflection from the repulsive part of the potential.

In Fig. 3(b) we present the outcome of the normalized $W\left(v_{z}^{\prime}, v_{z}\right)$ at $v_{z}^{\prime}=0$ for the cases of (i) small-angle quantum-mechanical hard-sphere scattering (dotted line), (ii) classical Lennard-Jones $(12,6)$ potential scattering using $(d \sigma / d \Omega)^{\mathrm{CLJ}}(\theta)$ (dashed line), and (iii) LJ $(12,6)$ potential scattering using the unified expression
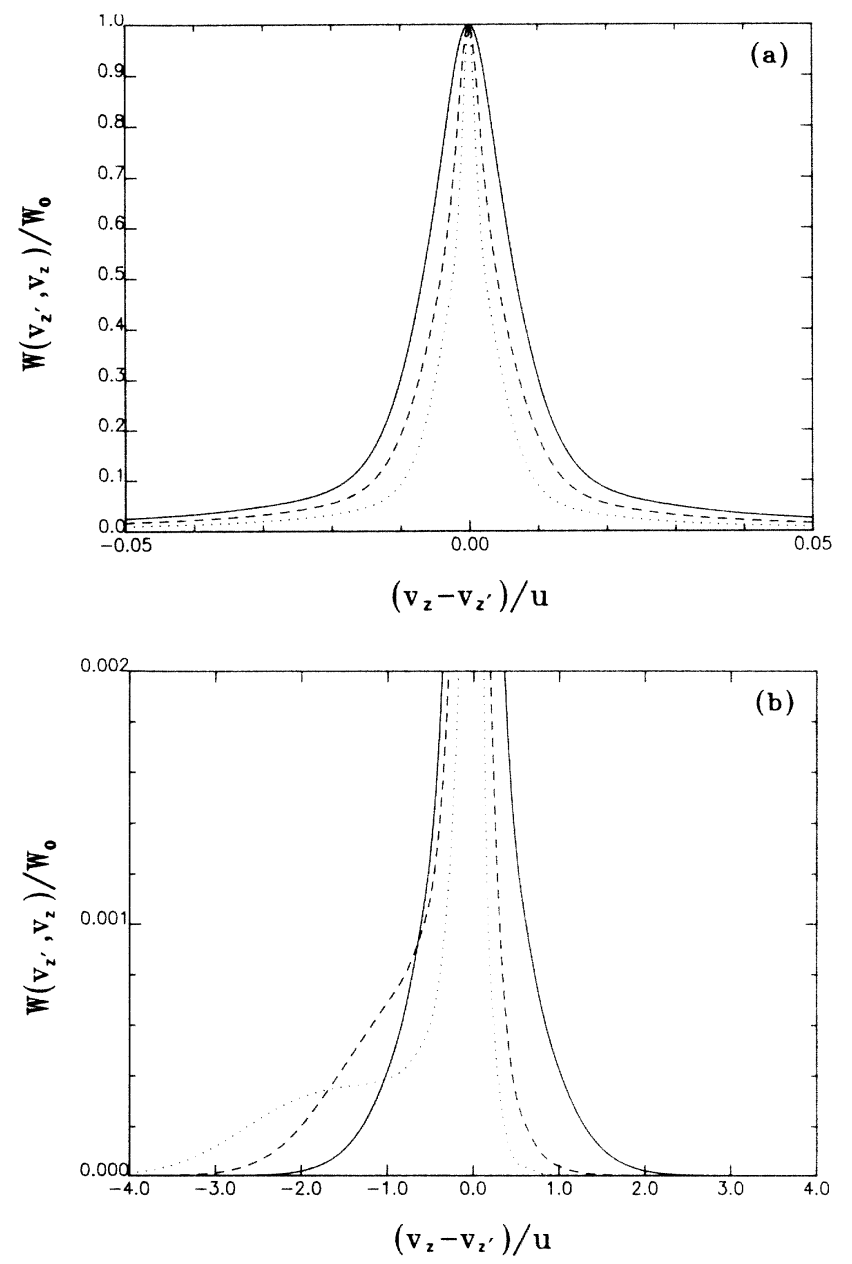

FIG. 4. (a) Normalized SCLJ one-dimensional collision kernel $W\left(v_{z}^{\prime}, v_{z}\right) / W_{0}$ as functions of $\left(v_{z}-v_{z}^{\prime}\right) / u$ for the Na-Ar system at $T=300 \mathrm{~K}$ in the velocity range -0.05 $\leqq\left(v_{z}-v_{z}^{\prime}\right) / u \leqq 0.05$. Solid curve, $v_{z}^{\prime}=0$; dashed curve, $v_{z}^{\prime}=u$; dotted curve, $v_{z}^{\prime}=2 u$. $u=\left(2 k_{B} T / m_{\mathrm{Na}}\right)^{1 / 2}$. (b) Same as Fig. 4(a), but $\left|\left(v_{z}-v_{z}^{\prime}\right) / u\right| \leqq 4.0$. $(d \sigma / d \Omega)^{\operatorname{SCLJ}}(\theta)$ (solid line). The SCLJ collision kernel (solid line) reflects the fully quantum-mechanical result as $(d \sigma / d \Omega)^{\operatorname{SCLJ}}(\theta)$ provides general average behavior of its quantum-mechanical counterpart at all angles as previously shown. The SCLJ collision kernel possesses a width smaller than that of small-angle QMHS model but much larger than that of the CLJ kernel. This can already be seen from their individual $(d \sigma / d \Omega)$ shown in Fig. 1. In fact, the normalized $W\left(v_{z}^{\prime}, v_{z}\right)$ corresponding to CLJ model should be infinitesimally narrow at $v_{z}^{\prime}=v_{z}$ because of the divergence of $(d \sigma / d \Omega)^{\mathrm{CLJ}}(\theta)$ at the forward direction $\theta=0$. (The finite width in CLJ collision kernel arises from the use of a finite number of mesh points in numerical integration.) In all three cases, we have found that (not shown) the collision kernels grow narrower, as $v_{z}^{\prime}$ departs more from zero, but all maximize at $v_{z}^{\prime}=v_{z}$. The latter result is quite different from the prediction of a displaced Gaussian line profile in the phenomenological Keilson-Storer ansatz.

The absolute magnitudes of $W\left(v_{z}^{\prime}, v_{z}\right)$ should be also noted. At $v_{z}=v_{z}^{\prime}=0$, for example, the ratio $W_{\text {SCLJ }}(0,0): W_{\text {QMHS }}(0,0): W_{\text {CHS }}(0,0)$ for $\mathrm{Na}-\mathrm{Ar}$ is about
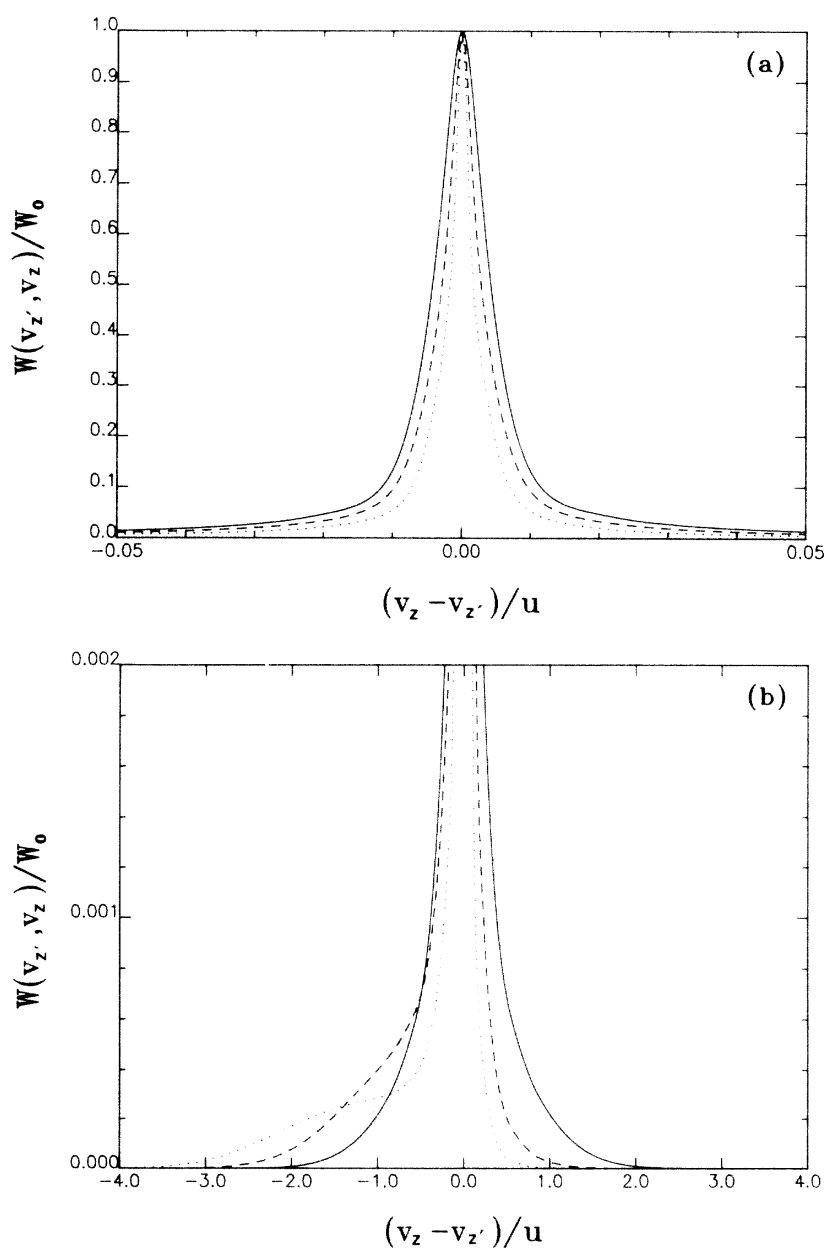

FIG. 5. Normalized SCLJ one-dimensional collision kernel $\boldsymbol{W}\left(v_{z}^{\prime}, v_{z}\right) / \boldsymbol{W}_{0}$ as functions of $\left(v_{z}-v_{z}^{\prime}\right) / u$ for the Ar-Ar system at $T=600 \mathrm{~K}$ where $u=\left(2 k_{B} T / m_{\mathrm{Ar}}\right)^{1 / 2}$. Curve notations of (a) and (b) same as Figs. 4(a) and 4(b). 
1:0.075:0.0018. That is, the absolute magnitudes of $W\left(v_{z}^{\prime}, v_{z}\right)$ from hard-sphere models can be several orders of magnitude smaller than the correct values. Similar conclusions are found for the Ar-Ar system.

In the rest of this section, we shall confine our discussion to the SCLJ collision kernel. The dependence of this collision kernel upon $v_{z}$ at $v_{z}^{\prime}=0, u$, and $2 u$ is shown in Fig. 4(a) nearby the resonant velocity, i.e., $\left|\left(v_{z}-v_{z}^{\prime}\right) / u\right| \leqq 0.05$, and in Fig. 4(b) for more extensive region (including the line wing), i.e., $\left|\left(v_{z}-v_{z}^{\prime}\right) / u\right| \leqq 4.0$, for the Na-Ar system. In the region around $v_{z}=v_{z}^{\prime}$ [Fig. 4(a)], i.e., corresponding to small-angle scatterings caused by the attractive part of the potential, the line profile of the normalized $W\left(v_{z}^{\prime}, v_{z}\right)$ becomes narrower as $v_{z}^{\prime}$ increases. This narrowing phenomenon is a manifestation of the fractional increase of the atoms being scattered into the small angles when the $z$ component of the atomic velocity increases. When $v_{z}^{\prime}$ departs from zero, the line profile exhibits asymmetry, with the left side $\left(v_{z}<v_{z}^{\prime}\right)$ profile being higher than that on the right side $\left(v_{z}>v_{z}^{\prime}\right)$. This asymmetry is due to the large-angle scattering effects caused by the repulsive potential, and is more clearly seen away from the resonance velocity region where the smallangle effects diminish, see Fig. 4(b). It is demonstrated in Fig. 4(b) that as $v_{z}^{\prime}$ increases, a shoulder gradually appears on the left side, whereas its counterpart on the right side is rapidly diminishing. This feature of the growth of the asymmetry has also been seen in the classical hard-sphere model in Fig. 3(a), and the order of magnitude of the shoulder is about the same for both the SCLJ and CHS calculations. This justifies the qualitative usefulness of the CHS model in the wing region.

Figure 5 shows the normalized SCLJ $W\left(v_{z}^{\prime}, v_{z}\right)$ profiles for the Ar-Ar system. In general, they exhibit the same properties as discussed for the $\mathrm{Na}-\mathrm{Ar}$ case. The potential well depth and the equilibrium position of the LJ potential and the relative mass ratio of the active atom and the perturber do not seem to have significant effect on the outlook of the elastic collision kernel under study.

\section{CONCLUSIONS}

In summary, we have studied the influence of the interatomic potentials and their approximate forms of the elastic differential cross sections on the one-dimensional elastic collision kernel $W\left(v_{z}^{\prime}, v_{z}\right)$, which is intimately relevant to the velocity-selective laser spectroscopy. Our analysis shows that the absolute magnitude of the collision kernel $W\left(v_{z}^{\prime}, v_{z}\right)$ is very sensitive while the normalized collision kernel $W\left(v_{z}^{\prime}, v_{z}\right)$ is moderately sensitive to the potential function used. This indicates the importance of employing realistic interatomic potential and therefore correct differential cross section at all angles in the accurate determination of the corresponding collision kernel. In the case of the Lennard-Jones $(12,6)$ potential, the SCLJ analytical model represents the correct average quantum-mechanical behavior of the elastic differential cross section for all angular range and serves as a good starting point for efficient evaluation of the collision kernel. The general properties of the collision kernel of the Lennard-Jones potential are thus correctly demonstrated, and many ambiguities associated with the drawbacks of the hard-sphere model, the phenomenological KeilsonStorer model, the small-angle classical long-range model, and the classical Lennard-Jones model are therefore clarified.

\section{ACKNOWLEDGMENTS}

This work was initiated while one of us (S.I.C.) was visiting the Joint Institute for Laboratory Astrophysics (JILA). He would like to thank Alan Gallagher for bringing his attention to the problem of velocity changing and for instructive discussions. This research was supported in part by the Department of Energy, Division of Chemical Sciences, and by the JILA Visiting Fellow Program.
${ }^{1}$ For reviews see P. R. Berman, Adv. At. Mol. Phys. 13, 57 (1977); Phys. Rep. 43, 101 (1978).

2J.-L. Le Goüet, J. Phys. B 11, 3001 (1978).

${ }^{3}$ Ph. Cahuzac, E. Marié, O. Robaux, R. Vetter, and P. R. Berman, J. Phys. B 11, 645 (1978); M. Gorlicki, A. Peuriot, and M. Dumont, J. Phys. (Paris) Lett. 41, L275 (1980).

${ }^{4}$ P. F. Liao, J. E. Bjorkholm, and P. R. Berman, Phys. Rev. A 21, 1927 (1980).

${ }^{5}$ P. R. Berman, T. W. Mossberg, and S. R. Hartmann, Phys. Rev. A 25, 2550 (1982).

${ }^{6}$ C. G. Aminoff, J. Javanainen, and M. Kaivola, Phys. Rev. A 28, 722 (1983).

${ }^{7}$ M. Gorlicki, C. Lerminiaux, and M. Dumont, Phys. Rev. Lett. 49, 1394 (1982).

${ }^{8}$ R. A. Forber, L. Spinelli, J. E. Thomas, and M. S. Feld, Phys. Rev. Lett. 50, 331 (1982).

9J.-C. Keller and J.-L. Le Goüet, Phys. Rev. Lett. 52, 2034 (1984); Phys. Rev. A 32, 1624 (1985).
10J. Keilson and J. E. Storer, Q. Appl. Math. 10, 243 (1952).

${ }^{11}$ M. Borenstein and W. E. Lamb, Jr., Phys. Rev. A 5, 1311 (1972).

${ }^{12}$ C. Bréchignac, R. Vetter, and P. R. Berman, J. Phys. (Paris) Lett. 39, L231 (1978).

${ }^{13}$ See, for example, M. S. Child, Molecular Collision Theory (Academic, London, 1974), Chap. 3.

${ }^{14}$ H. Pauly, Atom-Molecule Collision Theory, edited by R. B. Bernstein (Plenum, New York, 1979), Chap. 4.

${ }^{15}$ H. C. W. Beijerinck, P. M. A. Van Der Kam, W. J. G. Thidseen, and N. F. Verster, Chem. Phys. 45, 225 (1980).

${ }^{16}$ Accurate and rapid calculation of the phase shift $\delta_{l}$ in Eq. (7) can be achieved by using the Gauss-Mehler quadrature of a WKB integral, see, for example, R. T. Pack, J. Chem. Phys. 60, 633 (1974).

${ }^{17}$ R. Düren, Adv. At. Mol. Phys. 16, 55 (1980).

${ }^{18}$ R. A. Aziz, J. Chem. Phys. 65, 490 (1976); also Table 3 in Ref. 15. 